\title{
Successful Colistin Treatment of Multidrug-Resistant Pseudomonas aeruginosa Infection Using a Rapid Method for Determination of Colistin in Plasma: Usefulness of Therapeutic Drug Monitoring
}

\author{
Takehiro Yamada ${ }^{a, b}$ Nobuhisa Ishiguro, ${ }^{b}$ Kenji Oku, ${ }^{c}$ Issei Higuchi, ${ }^{a}$ Ikuma Nakagawa, ${ }^{c}$ \\ Atsushi Noguchi, ${ }^{c}$ Shinsuke Yasuda, ${ }^{c}$ Tatsuya Fukumoto,${ }^{b}$ Sumio Iwasaki, ${ }^{b}$ Kouji Akizawa, ${ }^{a}$ \\ Ayako Furugen, ${ }^{a}$ Hiroaki Yamaguchi, ${ }^{a}$ and Ken Iseki*,a,d \\ ${ }^{a}$ Department of Pharmacy, Hokkaido University Hospital; Kita 14, Nishi 5, Kita-ku, Sapporo 060-8648, Japan: \\ ${ }^{b}$ Infection Control Team, Hokkaido University; Kita 14, Nishi 5, Kita-ku, Sapporo 060-8648, Japan: ${ }^{c}$ Division of \\ Rheumatology, Endocrinology, and Nephrology, Hokkaido University Graduate School of Medicine; Kita 15, Nishi \\ 7, Kita-ku, Sapporo 060-8638, Japan: and ${ }^{d}$ Laboratory of Clinical Pharmaceutics and Therapeutics, Division of \\ Pharmasciences, Faculty of Pharmaceutical Sciences, Hokkaido University; Kita 12 Nishi 6, Kita-ku, Sapporo \\ 060-0812, Japan.
}

Received April 10, 2015; accepted June 23, 2015; advance publication released online July 9, 2015

A 56-year-old woman with systemic lupus erythematosus had bacteremia due to multidrug-resistant Pseudomonas aeruginosa (MDRP). She was initially treated with imipenem-cilastatin, tobramycin, and aztreonam; however, MDRP was still detected intermittently in her plasma. Multidrug-susceptibility tests demonstrated that MDRP was susceptible only to colistin. Therefore, in addition to these antibiotics, the administration of intravenous colistin methanesulfonate, a prodrug formula of colistin, was started at a daily dose of $2.5 \mathrm{mg} / \mathrm{kg}$ (as colistin base activity). The initial dose setting was based on the patient's renal function (baseline creatinine clearance $=32.7 \mathrm{~mL} / \mathrm{min}$ ). After initiating colistin, the patient's C-reactive protein levels gradually decreased. Blood cultures showed no evidence of MDRP on days 8, 14, and 22 after colistin initiation. However, the patient's renal function went from bad to worse owing to septic shock induced by methicillin-resistant Staphylococcus aureus (MRSA) infection. A few days later, the trough plasma levels of colistin were $7.88 \mathrm{mg} / \mathrm{L}$, which appeared to be higher than expected. After decreasing the colistin dose, the patient's renal function gradually improved. On the final day of colistin treatment, the plasma levels decreased to $0.60 \mathrm{mg} / \mathrm{L}$. MDRP could not be detected in blood culture after colistin treatment. Therefore, we successfully treated a case of bloodstream infection due to MDRP by therapeutic drug monitoring (TDM) of colistin. It is suggested that the monitoring of blood colistin levels by liquid chromatography-tandem mass spectrometry can contribute to safer, more effective antimicrobial therapy of MDRP because TDM facilitates quick decisions on dose adjustments.

Key words colistin; drug monitoring; multidrug-resistant Pseudomonas aeruginosa

Colistin, known as an "old" antibiotic, has recently been reintroduced in clinical practice ${ }^{1-3)}$ because of the increased emergence of infections caused by multidrug-resistant Gramnegative bacteria. ${ }^{2,4)}$ Colistin was originally developed in Japan in the 1940s-1950s; however, initial studies reported adverse reactions, including nephrotoxicity or peripheral nervous system disorders. ${ }^{5)}$ Because of these adverse events and the introduction of safer antibiotics, clinical use of colistin was abandoned in Japan in 1990.

On the other hand, some reports have indicated the usefulness of colistin against multidrug-resistant Pseudomonas aeruginosa (MDRP) infection., ${ }^{2,3)}$

Therapeutic drug monitoring (TDM) is a method used for determining the blood concentrations of drugs and optimizing the dose. Although a few case reports ${ }^{6,7)}$ have been published on TDM of colistin, these data were retrospectively determined after the end of treatment. To the best of our knowledge, this is the first report on a case in which TDM-based dosing optimization of colistin was applied to treat an MDRP bloodstream infection.

As for the determination method of drug level, traditional determination systems using high-performance liquid chromatography (HPLC) require time-consuming sample pre- treatment such as solid phase extraction and derivatization of colistin because of its poor UV absorption and no native fluorescence. ${ }^{8,9)}$ For the detection of colistin level in blood, we utilized liquid chromatography-tandem mass spectrometry (LC-MS/MS), a widely used technology for determination of drug concentrations because of its high sensitivity and selectivity, which enabled us to monitor and maintain optimal blood colistin levels. The retention time of colistin on HPLC and LC-MS/MS is $15 \mathrm{~min}^{9)}$ and $3 \mathrm{~min}$, respectively. Furthermore, it requires relatively little time to detect colistin. Prompt monitoring to ensure appropriate plasma colistin levels will contribute to more favorable treatment because patients suffering from MDRP infections are at a risk of rapid deterioration and thus require early treatment. Here we report a case in which colistin dose was carefully adjusted using TDM.

\section{PATIENTS AND METHODS}

Plasma Colistin Determination Plasma colistin levels were determined using LC-MS/MS using the method reported by Jansson et al. ${ }^{10)}$ with slight modification. In brief, $180 \mu \mathrm{L}$ of plasma was spiked with $20 \mu \mathrm{L}$ of colistin working solution $(100,50,25,12.5$, and $0 \mathrm{mg} / \mathrm{L})$, and polymyxin B was used as 

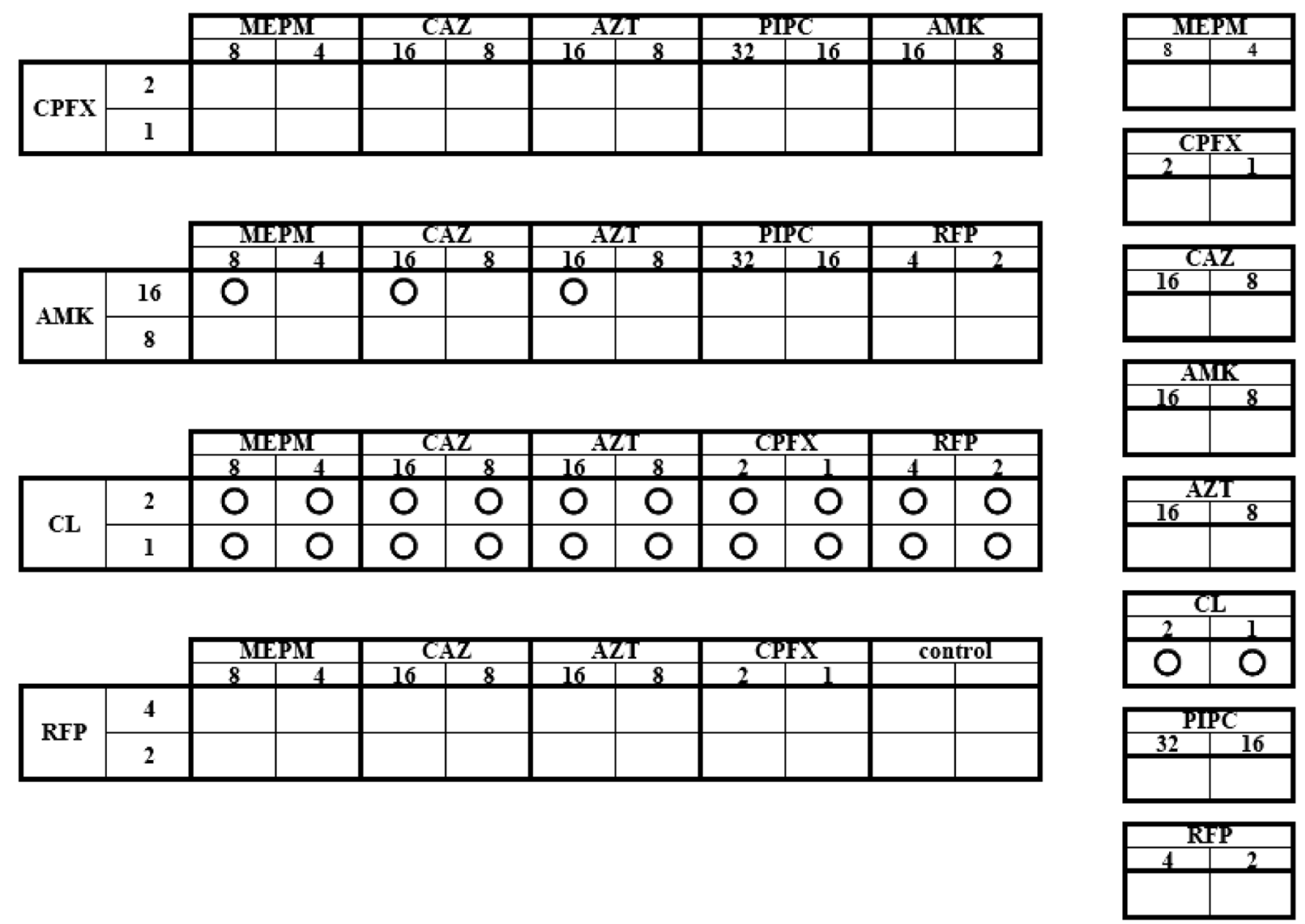

Fig. 1. Investigation of the Synergistic Effects of Antibiotics with Colistin Using the Breakpoint Checkerboard Plate

This assay is usually performed to evaluate the in vitro effects of the combination of multiple antibiotics against isolated MDRP in blood from this patient. Numbers mean concentration $(\mathrm{mg} / \mathrm{L})$ of each antibiotics which showed inhibitory effects against isolated strain. MEPM, meropenem; CAZ, ceftazidime; AZT, aztreonam; PIPC, piperacillin; AMK, amikacin; RFP, rifampicin; CPFX, ciprofloxacin; CL, colistin.

the internal standard. The samples were mixed with equal volumes of $0.1 \%$ trifluoroacetic acid in acetonitrile. After centrifugation at $15000 \mathrm{rpm}$ for $15 \mathrm{~min}$, the supernatant was mixed with an equal volume of $0.03 \%$ trifluoroacetic acid in water. Aliquots of the mixture $(30 \mu \mathrm{L})$ were applied to LC-MS/MS.

Ethics The present study was carried out in accordance with the guidelines for the care for human study, and the study protocol was approved by the ethics committee of the Hokkaido University Hospital.

\section{CASE REPORT}

A 56-year-old woman (body height, $150 \mathrm{~cm}$ and weight, $61.4 \mathrm{~kg}$ ) with systemic lupus erythematosus had bacteremia due to MDRP. She had been treated with imipenem-cilastatin (IPM/CS; $500 \mathrm{mg}$, twice daily) against extended-spectrum beta-lactamase-producing Krebsiella pneumoniae, tobramycin (TOB; $2 \mathrm{mg} / \mathrm{kg}$, every $48 \mathrm{~h}$ ), which was the only sensitive antibiotic for initially detected Pseudomonas aeruginosa, and aztreonam (AZT; $1000 \mathrm{mg}$, twice daily) for $57 \mathrm{~d}$; however, MDRP was still detected in blood intermittently. On the other hands, before detection in blood culture, MDRP was detected in a urine sample ( $62 \mathrm{~d}$ before the start of colistin treatment), and the patient presented with pyelonephritis due to the bacterial infection. Thus, the source of infection was thought to be from urinary tract in this case. Multidrug susceptibility tests using the breakpoint checkerboard plate method demonstrated that MDRP was susceptible only to colistin, whose minimum inhibitory concentration against MDRP was equal to or less than $1 \mathrm{mg} / \mathrm{L}$ (Fig. 1). Therefore, in addition to AZT, intravenous colistin (as a prodrug formula) was initiated at a daily dose of $2.5 \mathrm{mg} / \mathrm{kg}$ (as colistin base activity, Fig. 2). The initial dose setting of colistin was based on the guidelines of the Japanese Society of Chemotherapy for the appropriate use of colistin (released on July 2012). Prior to the administration of colistin, $N$-acetyl- $\beta$-D-glucosaminidase (NAG) levels in urine were $39.4 \mathrm{U} / \mathrm{L}$ and the urinary creatinine-adjusted value (NAG/Cre) was 158. Eight days after starting colistin, NAG and NAG/Cre levels were markedly elevated to $75.3 \mathrm{U} / \mathrm{L}$ and 290, respectively (Fig. 3). On the first day of colistin administration, serum creatinine levels and creatinine clearance $(\mathrm{CCr}$, calculated using the Cockcroft-Gault equation) were $1.86 \mathrm{mg}$ / $\mathrm{dL}$ and $32.7 \mathrm{~mL} / \mathrm{min}$, respectively. Until day 14 after initiating colistin, serum creatinine levels increased gradually to $2.77 \mathrm{mg} / \mathrm{dL}$ and $\mathrm{CCr}$ was $23.6 \mathrm{~mL} / \mathrm{min}$. On day 13 , the trough levels of colistin were found to be higher $(7.88 \mathrm{mg} / \mathrm{L})$ than expected $^{1)}$ (Fig. 3). Therefore, the daily dosage of colistin was decreased to $1.5 \mathrm{mg} / \mathrm{kg}$; however, the patient's serum creatinine levels remained high for approximately $48 \mathrm{~h}$, after which her renal function recovered, showing serum creatinine levels of $1.36 \mathrm{mg} / \mathrm{dL}$ on the day after decreasing colistin dosage (day 20, Fig. 2). On days 8, 14 and 22 after initiating colistin, MDRP could not be detected in her blood. Moreover, MDRP could not be detected in the blood culture even one week after the end of colistin treatment. Colistin was continued for $21 \mathrm{~d}$ and the C-reactive protein (CRP) levels markedly decreased from 13.75 to $2.47 \mathrm{mg} / \mathrm{dL}$. Overall, the colistin treatment was clinically and bacteriologically effective. Meanwhile, $10 \mathrm{~d}$ 


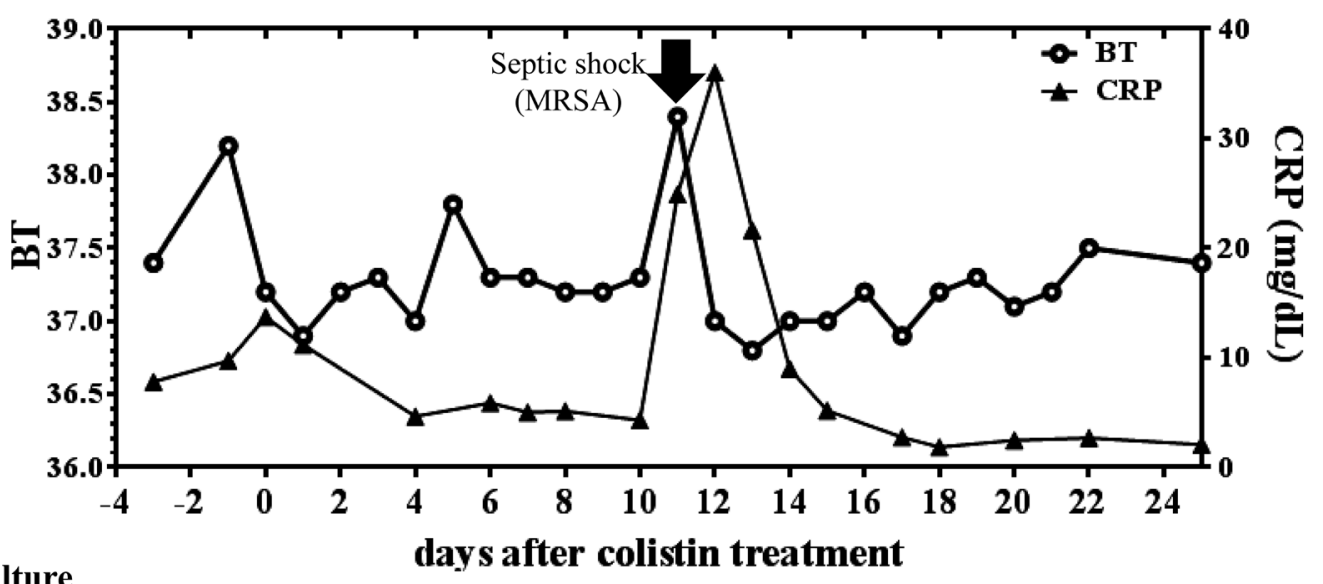

Blood culture

\begin{tabular}{lllll}
\hline MDRP & $(+)(+)$ & $(-)$ & $(-)$ & $(-)$ \\
\hline MRSA & $(+)(+)$ & $(-)$ & $(-)$ \\
\hline
\end{tabular}

\begin{tabular}{cr|}
\cline { 2 - 2 } CL & $2.5 \mathrm{mg} / \mathrm{kg} \mathrm{q24h}$ \\
AZT & $2000 \mathrm{mg} \mathrm{q24h}$ \\
\cline { 2 - 2 } CAZ & $1.5 \mathrm{mg} / \mathrm{kg} \mathrm{q48h}$ \\
\cline { 2 - 2 } DAP & $1000 \mathrm{mg} \mathrm{q24h}$ \\
& $5.7 \mathrm{mg} / \mathrm{kg} \mathrm{q48h}$ \\
\hline
\end{tabular}

Fig. 2. Clinical Course of This Case

Colistin was initiated against blood-stream infection of MDRP. After starting colistin, MDRP in blood was disappeared on the day 8 . Although this patient fell into septic shock evoked by MRSA infection (day 11, arrow), which was improved after starting daptomycin (DAP). BT, body temperature; CRP, C-reactive protein.

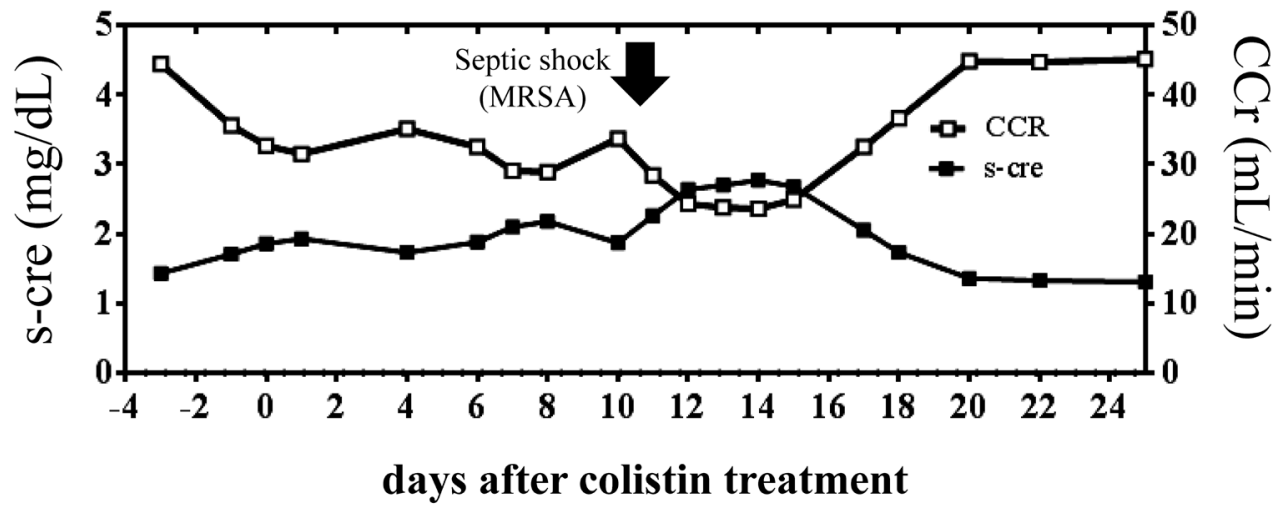

\begin{tabular}{|c|c|c|c|}
\hline NAG/CRE & 158 & 290 & \\
\hline $\begin{array}{l}\text { colistin }(\mathbf{m g} / \mathbf{L}) \\
\text { trough level }\end{array}$ & & 7.88 & 0.60 \\
\hline
\end{tabular}

Fig. 3. Change of Renal Function and Plasma Colistin Level during the Antimicrobial Treatment

Renal function got worse after MRSA-evoked septic shock (day 11, arrow). NAG, $N$-acetyl- $\beta$-D-glucosaminidase; CRE, creatinine; s-cre, serum creatinine; CCr, creatinine clearance.

after starting colistin, the patient fell into septic shock owing to methicillin-resistant Staphylococcus aureus (MRSA) infection. A subcutaneous abscess caused by MRSA under the sutured wound because of the aortic valve replacement after developing congestive heart failure was thought to be the source of MRSA sepsis. On this day, ceftazidime was started for the reinforcement of the treatment against the Gram-negative bacterial infection. After starting daptomycin (DAP), CRP and BT levels decreased markedly (Fig. 2). On the last day of colistin treatment, her BT and CRP levels had improved and her renal function recovered to nearly normal levels.

\section{DISCUSSION}

The emergence of MDRP is becoming one of the major clinical issues in nosocomial infections, particularly in criti- 
cally ill patients. Colistin is considered the first line drug for MDRP but has not yet been approved in Japan. We therefore obtained a parenteral formula of colistin [as the prodrug colistin methanesulfonate (CMS)] from a pharmaceutical company outside Japan. Recently, Mizuyachi et al. ${ }^{11)}$ reported the pharmacokinetic profiles of colistin and CMS in healthy Japanese male subjects, and Couet et al. ${ }^{12)}$ reported the results in healthy Caucasian subjects. The plasma half-life $\left(t_{1 / 2}\right)$ of colistin is estimated to be approximately $3 \mathrm{~h}$. However, in a study of critically ill patients, $t_{1 / 2}$ of colistin was prolonged up to $14.4 \mathrm{~h} .{ }^{1)}$ In the previous study, a strong inverse trend was observed between steady-state plasma colistin levels and creatinine clearance. Thus, renal dosage and interval adjustment in colistin treatment are required, particularly for patients with kidney dysfunction.

A prospective observational cohort study demonstrated that trough plasma levels of colistin is an independent risk factor for nephrotoxicity ${ }^{13)}$ and that trough plasma colistin levels of $3.33 \mathrm{mg} / \mathrm{L}$ on day 7 best predicted acute kidney injury (AKI). Information on the plasma levels of colistin will be helpful for future dosing recommendations. To promptly determine the colistin trough level, we utilized a new method based on LC-MS/MS without time-consuming preparation. We experienced trough colistin levels as high as $7.88 \mathrm{mg} / \mathrm{L}$ on day 13 after starting colistin treatment. The patient's renal function tended to worsen, with creatinine clearance decreasing to $23.8 \mathrm{~mL} / \mathrm{min}$ because of sepsis evoked by MRSA bloodstream infection; however, this transient renal dysfunction may have been partly due to colistin.

From day 14 after initiation of DAP for MRSA-induced sepsis, the patient's renal function recovered gradually. On day 20 after starting colistin treatment, plasma colistin levels were decreased by $0.61 \mathrm{mg} / \mathrm{L}$; this decline was thought to be due to the recovery of her creatinine clearance.

If the plasma levels of colistin are below the MIC value for a very large proportion of the dosage interval, there is a possibility that the treatment has failed. ${ }^{8)}$ In the present case, MDRP in the blood culture was negative even one week after the end of colistin treatment. Her CRP level was remained lower than that on the day of starting colistin treatment, suggesting bacteriological and clinical effectiveness of colistin in this case. In addition, although the NAG/CRE value was examined only 2 points (Fig. 2), her daily urinary volume recovered to normal level (day 13 of colistin treatment, $150 \mathrm{~mL}$, day $20,2200 \mathrm{~mL}$ ) after decreasing the colistin daily dose, suggesting that dose optimization of colistin is effective. These effects were due to TDM-based dosing of colistin, which contribute to avoid worsening of the renal function. Thus, TDMbased dosing of colistin is beneficial for patient safety. We were able to use colistin in a safer and more effective manner with TDM than without it. TDM-based colistin therapy will be a strong method for the patients suffering from MDRP.

Conflict of Interest The authors declare no conflict of interest.

\section{REFERENCES}

1) Garonzik SM, Li J, Thamlikitkul V, Paterson DL, Shoham S, Jacob J, Silveira FP, Forrest A, Nation RL. Population pharmacokinetics of colistin methanesulfonate and formed colistin in critically ill patients from a multicenter study provide dosing suggestions for various categories of patients. Antimicrob. Agents Chemother., 55, 3284-3294 (2011).

2) Li J, Nation RL, Turnidge JD, Milne RW, Coulthard K, Rayner CR, Paterson DL. Colistin: the re-emerging antibiotic for multidrugresistant Gram-negative bacterial infections. Lancet Infect. Dis., 6, 589-601 (2006).

3) Martis N, Leroy S, Blanc V. Colistin in multi-drug resistant Pseudomonas aeruginosa blood-stream infections: a narrative review for the clinician. J. Infect., 69, 1-12 (2014).

4) Montero M, Horcajada JP, Sorli L, Alvarez-Lerma F, Grau S, Riu M, Sala M, Knobel H. Effectiveness and safety of colistin for the treatment of multidrug-resistant Pseudomonas aeruginosa infections. Infection, 37, 461-465 (2009).

5) Falagas ME, Kasiakou SK, Saravolatz LD. Colistin: the revival of polymyxins for management of multidrug-resistant Gram-negative bacterial infections. Clin. Infect. Dis., 40, 1333-1341 (2005). Corrected in Clin. Infect. Dis. in 2006, 42, 1819.

6) Yaita K, Sameshima I, Takeyama H, Matsuyama S, Nagahara C, Hashiguchi R, Moronaga Y, Tottori N, Komatsu M, Oshiro Y, Yamaguchi Y. Liver abscess caused by multidrug-resistant Pseudomonas aeruginosa treated with colistin; a case report and review of the literature. Intern. Med., 52, 1407-1412 (2013).

7) Bode-Böger SM, Schopp B, Tröger U, Martens-Lobenhoffer J, Kalousis K, Mailänder P. Intravenous colistin in a patient with serious burns and borderline syndrome: the benefits of therapeutic drug monitoring. Int. J. Antimicrob. Agents, 42, 357-360 (2013).

8) Li J, Coulthard K, Milne R, Nation RL, Conway S, Peckham D, Etherington C, Turnidge J. Steady-state pharmacokinetics of intravenous colistin methanesulphonate in patients with cystic fibrosis. $J$. Antimicrob. Chemother., 52, 987-992 (2003).

9) Li J, Milne RW, Nation RL, Turnidge JD, Coulthard K, Valentine J. Simple method for assaying colistin methanesulfonate in plasma and urine using high-performance liquid chromatography. Antimicrob. Agents Chemother., 46, 3304-3307 (2002).

10) Jansson B, Karvanen M, Cars O, Plachouras D, Friberg LE. Quantitative analysis of colistin A and colistin B in plasma and culture medium using a simple precipitation step followed by LC-MS/MS. J. Pharm. Biomed. Anal., 49, 760-767 (2009).

11) Mizuyachi K, Hara K, Wakamatsu A, Nohda S, Hirama T. Safety and pharmacokinetic evaluation of intravenous colistin methansulfonate sodium in Japanese healthy male subjects. Curr. Med. Res. Opin., 27, 2261-2270 (2011). Corrected in Curr. Med. Res. Opin., 2015, 31, 593-594.

12) Couet W, Gregoire N, Gobin P, Saulnier PJ, Frasca D, Marchand S, Mimoz O. Pharmacokinetics of colistin and colistimethate sodium after a single 80 -mg intravenous dose of CMS in young healthy volunteers. Clin. Pharmacol. Ther., 89, 875-879 (2011).

13) Sorli L, Luque S, Grau S, Berenguer N, Segura C, Montero MM, Alvarez-Lerma F, Knobel H, Benito N, Horcajada JP. Trough colistin plasma level is an independent risk factor for nephrotoxicity: a prospective observational cohort study. BMC Infect. Dis., 13, 380 (2013). 